\title{
Multi-objective genetic algorithm optimization of linear proportional solenoid actuator
}

\author{
Shi Jie Wang ${ }^{1} \cdot$ Zhi Dan Weng $^{2} \cdot$ Bo Jin $^{1,3}$ (1) $\cdot$ Hong Xu Cai ${ }^{1}$
}

Received: 8 September 2019 / Accepted: 7 December 2020 / Published online: 15 January 2021

(c) The Author(s) 2021

\begin{abstract}
Linear proportional solenoid (LPS) is widely applied in different linear motion control systems as the electromagnetic actuator since its high reliability and low cost. LPS is difficult to optimize by changing a single variable due to amounts of structural design parameters, and each design parameter has a nonlinear relationship with the static electromagnetic force. This paper aims to improve LPS's push force and response performance through magnetostatic finite element analysis (FEA) by ANSYS MAXWELL. This study compares FEA 2D model, 3D model and measurement results underrated coil current to verify the accuracy of FEA 2D model. In order to reveal the nonlinear relationship between shape design parameters and electromagnet design objectives, this study compares the influence degree of each variable on each design objective by conventional type LPS 2D FEA model. And for the purpose of improving LPS's push force and response performance, a multi-objective optimization method has been proposed in this study based on genetic algorithm (GA) and magnetostatic FEA 2D model for optimizing the shape design parameters. All the study results were validated in both static conditions and dynamic conditions. The comparison between manufactured optimal type and conventional type results shows that the static push force in working stroke is improved $30.1 \%$, displacement step response rise time is reduced $5.2 \%$ and $43.4 \%$, and force step response rise time is reduced $20.5 \%$ and $44.6 \%$ with different return spring stiffness. Above all, LPS static and dynamic performance has been improved directly and the validation of proposed optimization method is verified in this paper.
\end{abstract}

Keywords Proportional control valve $\cdot$ Solenoid actuator $\cdot$ Multi-objective optimization $\cdot$ Genetic algorithm

\section{Introduction}

As micro-processing units have been rapidly developed in recent year, the control methods in the traditional fluid control field have gradually shifted to electronic control.

Technical Editor: Victor Juliano De Negri.

Bo Jin

bjin@zju.edu.cn

Shi Jie Wang

911956918@qq.com

Zhi Dan Weng

derek.weng@hoyea.com

1 State Key Laboratory of Fluid Power and Mechatronic Systems, Zhejiang University, Hang Zhou 310027, China

2 Ningbo HOYEA Machinery Manufacture Co.Ltd., Ningbo 315131, China

3 Ningbo Research Institute, Zhejiang University, Ningbo 315100, China
Electromagnetic actuators are the core component of vehicle suspension system and engine, inverter compressor and many other pneumatic/hydraulic control systems. As a type of electromagnetic actuator, LPS is generally employed in proportional control valve (PCV) due to its simple structure, high reliability, low cost and long stroke. Therefore, the study of electromagnetic actuators is necessary for the improvement in electrohydraulic control performance.

In last decades, numerous publications about the performance improvement in electromagnetic actuators can been found in the literature. For variable valve timing technique of vehicle engine, a study in 2015 written by Lee, S.H et al. aim to reduce the transient time of strokes for higher engine speed by using the natural frequency as an optimal design goal [1]. For electronic fuel injection systems of fuel cell vehicle engine, temperature rise and electromagnetic force at saturation temperature are required for ultra-high pressure conditions in the published paper written by Lee et al. [2]. High-speed solenoid valve (HSV) is the key control unit of common-rail injector, and Zhao et al. aim to reduce the 
useless energy by decreasing the hold current of HSV in Ref. [3]. And a 3D FEA model is employed to analyze the power distribution under different hold current. The volume of electromagnetic actuators is also an important condition, and Cai et al. [4] optimize five main shape parameters to obtain the maximum electromagnetic force in a specific valve volume. Reference [5] aims at optimizing the performances of response time, size and thermal performance multi-objective optimization method. The optimization strategies of solenoid valve include geometry shape design [6], energy distribution optimization for power consumption [7] and electromechanical system parameters design [2]. The numerical magnetic field calculation methods include equivalent magnetic circuit (EMC) method and finite element analysis (FEA) method. And the optimization algorithms of optimization method include the parametric analysis method, gradient optimizations and global optimization algorithms.

Besides, the same dynamic property requirement of opening/closing time as On/Off electromechanical actuator, LPS requires a certain degree of proportionality between the proportional static electromagnetic force and coil exciting current, which would not change with the displacement of LPS in working stroke [8]. Thus, the performance optimization of LPS is a multi-objective design problem. As performance optimization objectives in this research, maximum static electromagnetic force, moving mass and minimum electromagnetic force displacement gradient in working stroke are employed to obtain an optimal design LPS, which can meet the requirements of large push force and quick response. An optimization strategy is proposed including excitation coil parameters, actuator geometry shape and electromechanical system parameters design. Based on ANSYS MAXWELL FEA methods, a shape optimization of LPS is performed in order to obtain high accuracy. Many parametric methods are used for the shape optimization of electromagnetic devices $[9,10]$, but the obvious disadvantages of parametric analysis methods could also be found. For example, it has a heavy workload of multi-objective for researcher and it usually obtains a local optimum result. Therefore, a global optimization algorithm genetic algorithm (GA) is really necessary for electromechanical solenoid actuator in current researches [11-13]. When using genetic algorithm (GA) optimization algorithm to optimize the actuator, using three-dimensional model might waste a lot of time in simulation. Considering the simulation time cost of the FEA method, the two-dimensional axial symmetry FEA model is more suitable for this research than the three-dimensional model.

In summary, the whole research would be introduced in the following chapters. First, it will explain the theoretical model of electromagnetic LPS, which is composed by two parts: the structure of electromagnetic LPS and electromagnetic mathematic model of LPS. Second, the optimization procedure of LPS will be described by the aforementioned method, and then, the optimum LPS prototype will be finalized and manufactured. Third, it will make a comparison between numerical magnetic field calculation and experimental results that could validate the improvement of proposed optimization procedure.

\section{Materials and methods}

\subsection{The structure of electromagnetic LPS}

The LPS is a push type solenoid electromagnetic actuator with specific non-magnetically conductive ring (NMR) structure. The LPS is also an axial symmetry shape, and the simulation model can be simplified and shown in Fig. 1a, which consists of shaft, stator, coil, coil frame, yoke, nonmagnetic ring, armature and return spring. Each part of LPS has several design variables, the definitions of shape design parameters are shown in Fig. 1b, and the design range of these parameters are shown in Table.1.

Except above design parameters, the interstice between armature and armature cover yoke can also be fixed for economical fabrication purpose. Conventionally, the interstice is $0.15 \mathrm{~mm}$ in radial direction to film Teflon non-magnetic conductive tap. This approach can reduce the armature moving friction.

\subsection{The electromagnetic mathematic model of LPS}

In both open-loop and close-loop control system, the input signal $(+10 \mathrm{~V} \sim-10 \mathrm{~V})$ is proportional to the LPS coil current. Additionally, for the reason of spring compression and other resistance force, the position of armature is proportional to the LPS coil current and the input signal. This linear characteristic of LPS will reduce the control complexity. Therefore, the main design purpose of LPS is to make sure the electromagnetic force is proportional to the coil current and is constant to the actuator displacement in operating stroke. Since LPS is an axial symmetry structure and the actuator axial electromagnetic force is a static design purpose, the Maxwell's equations will be simplified as follows:

The magnetic field strength $H$ is obtained by the Ampère's law

$H=\frac{I N}{\delta}$

where $I$ represents coil excitation current, $N$ is the number of turns of coil and $\delta$ represents the length of flux path.

According to the definition of magnetic flux- $\phi$ and magnetic flux density- $B$, expression (4) is obtained as follows:

$\phi=B S$

$B=\mu H$ 
Fig.1 (a) Structure of LPS and (b) parameters definitions of LPS

Table 1 Definitions of LPS shape design parameters

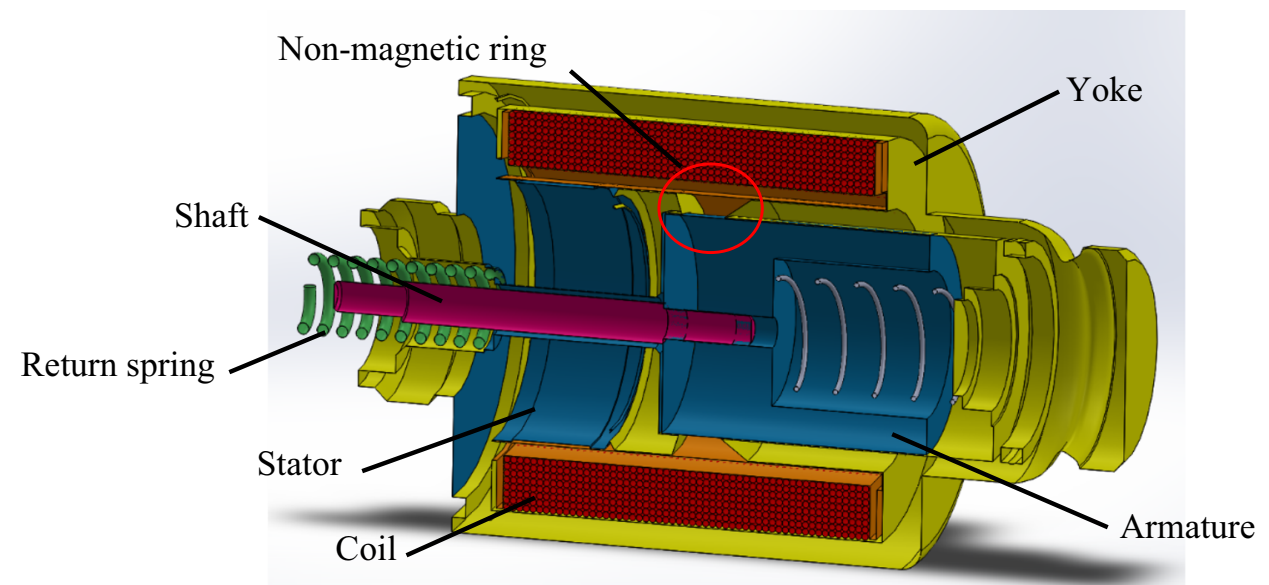

(a)

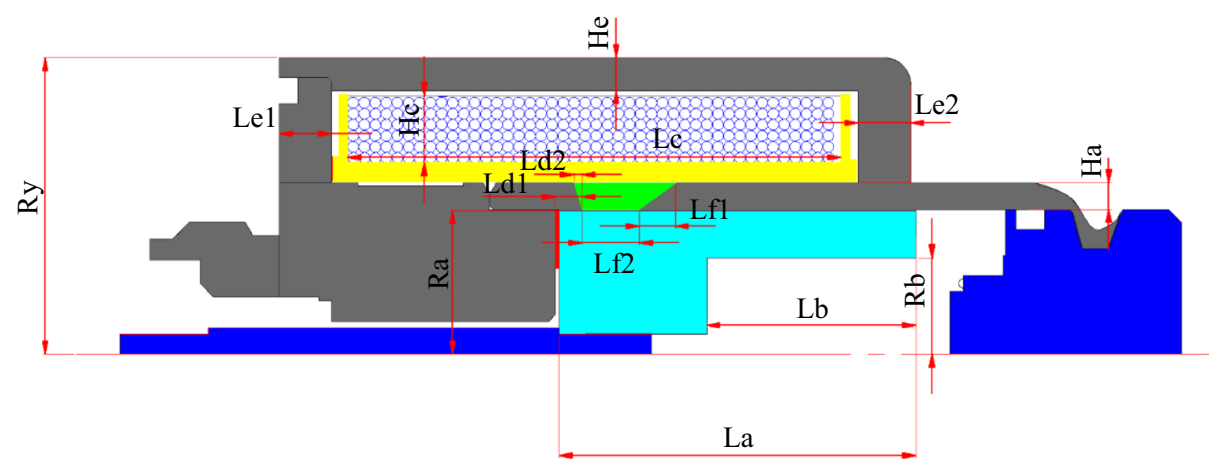

(b)

\begin{tabular}{lll}
\hline LPS design parameter & Symbol & \\
\hline Actuator radius & $\mathrm{Ry}$ & $22.5 \mathrm{~mm}$ (fixed) \\
Armature radius & $\mathrm{Ra}$ & Correlated with Ry, Ha, Hc, He \\
Spring bore radius & $\mathrm{Rb}$ & $5-9 \mathrm{~mm}$ \\
Armature length & $\mathrm{La}$ & $24-46 \mathrm{~mm}$ \\
Spring bore depth & $\mathrm{Lb}$ & $2 \sim 18 \mathrm{~mm}$ \\
Coil length & $\mathrm{Lc}$ & Correlated with Hc \\
NMR to stator distance & $\mathrm{Ld} 1$ & $1.9-2.9 \mathrm{~mm}$ \\
NMR front length & $\mathrm{Ld} 2$ & $0.1 \sim 2.4 \mathrm{~mm}$ \\
Front yoke thickness & $\mathrm{Le} 1$ & $4 \mathrm{~mm}(\mathrm{fixed})$ \\
Back yoke thickness & $\mathrm{Le} 2$ & $4 \mathrm{~mm}(\mathrm{fixed})$ \\
NMR back length & $\mathrm{Lf} 1$ & $1-7 \mathrm{~mm}$ \\
NMR length & $\mathrm{Lf} 2$ & $2.7-7.7 \mathrm{~mm}$ \\
Armature cover yoke thickness & $\mathrm{Ha}$ & $1.5-3.5 \mathrm{~mm}$ \\
Coil thickness & $\mathrm{Hc}$ & $4.5-6 \mathrm{~mm}$ \\
Coil cover yoke thickness & $\mathrm{He}$ & $2.5 \mathrm{~mm}$ (fixed)
\end{tabular}

$$
\phi=\frac{I N}{R_{\delta}}
$$

where $\mu$ is permeability. $R_{\delta}$ is the reluctance, the axial electromagnetic force of armature expression (5) [9] can be obtained as expression (6): 
$F_{x}=\frac{1}{2} \cdot \frac{B^{2} S}{\mu_{0}}$

$F_{x}=\frac{1}{2} \cdot \frac{I^{2} N^{2}}{\mu_{0} R_{\delta}^{2} S}$

where $\mu_{0}=4 \pi \times 10^{-7} \mathrm{H} / \mathrm{m}$ is the permeability of free space, and $S$ represent the cross sectional area of LPS's armature. Ignoring the reluctance of ferromagnetic material, the simplified magnetic flux line is shown in Fig. 2.

The shaft is fixed by the hole located in middle of the armature and the electromagnetic force of armature transferred to the valve spool by shaft. Thus, each magnetic flux line reluctance can be described as follows:

$R_{1}=\frac{x}{\mu_{0} \pi A_{a}}$

where $A_{a}$ is the effective area of armature head surface. $x$ is the displacement to the stator.

$R_{2}=\frac{\frac{\pi}{4}(d+z)}{\mu_{0} \pi R a^{2}-\mu_{0} \pi(R a+d-z)^{2}}, z=\frac{2 x}{\pi}$

$R_{3}=\frac{d}{\mu_{0} \pi(2 R a+2 d)(L d 1-x)}$

where $d$ is the interstice in radial direction between armature and armature cover yoke.

$R_{4}=\frac{d}{\mu_{0} \pi(2 R a+2 d)(L a+z-L d 1-L f 2)}$

Thus, the reluctance $R_{\delta}$ is:

$R_{\delta}=\frac{R_{1} R_{2} R_{3}}{R_{1} R_{2}+R_{1} R_{3}+R_{2} R_{3}}+R_{4}$

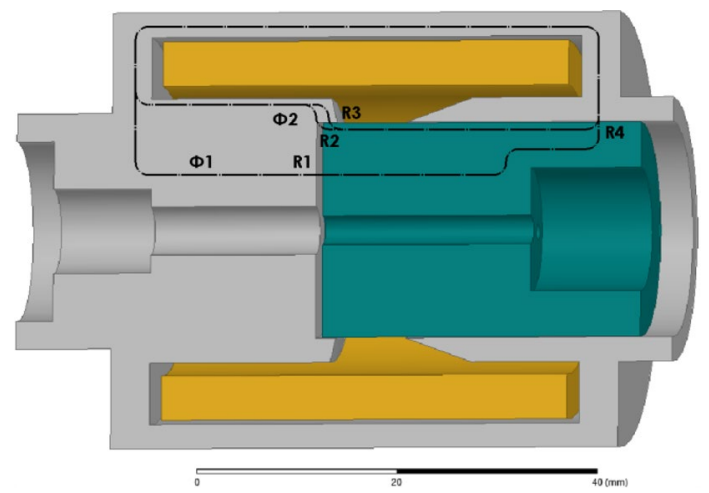

Fig.2 Simplified magnetic flux line in FEA 3D model
Consider Eqs. (6) and (11), the static axial electromagnetic force of armature and the coil current maintain a nonlinear relationship in different armature displacement. For the linear control requirement $f$ LPS, the shape design of LPS is an important process in LPS performance improvement.

Coil winding design is the first process in LPS optimization. The mathematic model of coil winding is described by following equations:

$N=\frac{f_{w} L c H c}{s_{w}}$

where $f_{w}$ is the winding stacking factor and $s_{w}$ is the wire cross section. The actuator design parameter $L c$ and $H c$ can be determined by the winding number $N, f_{w}$ and $s_{w}$. Based on practical experience, the winding stacking factor is fixed as 0.7 and $s_{w}$ less than $0.54 \mathrm{~mm}^{2}$. According to the cable maximum temperature and electronic control drivers power consumption, this paper will choose cable upper limit $s_{w}$ and set rated coil excitation current as $5.4 \mathrm{~A}$ for obtaining the less coil power consumption.

\section{Description of LPS FEA model and optimization process}

The FEA model of LPS is used to calculate the static electromagnetic force by ANSYS Electronics software Maxwell. LPS shape design GA optimization is employed to obtain the ideal static electromagnetic force-displacement curve and moving part mass.

\subsection{FEA model description}

In this study, 2D FEA model is applied to reduce the time cost of manufacture experiment. Therefore, the conventional shape LPS is applied in comparison test to validate the availability of 2D FEA model. Except some fixed parameter, all GA optimization design variables and values are presented in Table.2. And the static force versus displacement curves obtained from 2D FEA simulation, 3D FEA simulation and experiment result are shown in Fig. 3. These curves are underrated excitation current $2.4 \mathrm{~A}$ (total $1188 \mathrm{~A}$ turns). Through the comparison of those curves, Fig. 3 indicates that maximum deviation rate of 2D FEA model is $8.1 \%$, and 3D model is $7.1 \%$. Meanwhile, the comparison results also demonstrate that 2D FEA model has almost same simulation accuracy with 3D model in magnetostatic numerical calculation. Thus, both static force obtained by 2D FEA model and 3D FEA model showed good agreement with the measurement results and 2D FEA model has less time cost. 
Table 2 Value of LPS shape design variables

\begin{tabular}{ll}
\hline Symbol of parameter & Conventional value \\
\hline $\mathrm{Ra}$ & $9.35 \mathrm{~mm}$ \\
$\mathrm{Rb}$ & $6.75 \mathrm{~mm}$ \\
$\mathrm{La}$ & $27 \mathrm{~mm}$ \\
$\mathrm{Lb}$ & $7.3 \mathrm{~mm}$ \\
$\mathrm{Lc}$ & $40 \mathrm{~mm}$ \\
$\mathrm{Ld} 1$ & $2.38 \mathrm{~mm}$ \\
$\mathrm{Ld} 2$ & $1.15 \mathrm{~mm}$ \\
$\mathrm{Lf} 1$ & $0.72 \mathrm{~mm}$ \\
$\mathrm{Lf} 2$ & $5.2 \mathrm{~mm}$ \\
$\mathrm{Ha}$ & $2 \mathrm{~mm}$ \\
$\mathrm{Hc}$ & $6.75 \mathrm{~mm}$ \\
Armature mass & $46.2 \mathrm{~g}$ \\
\hline
\end{tabular}

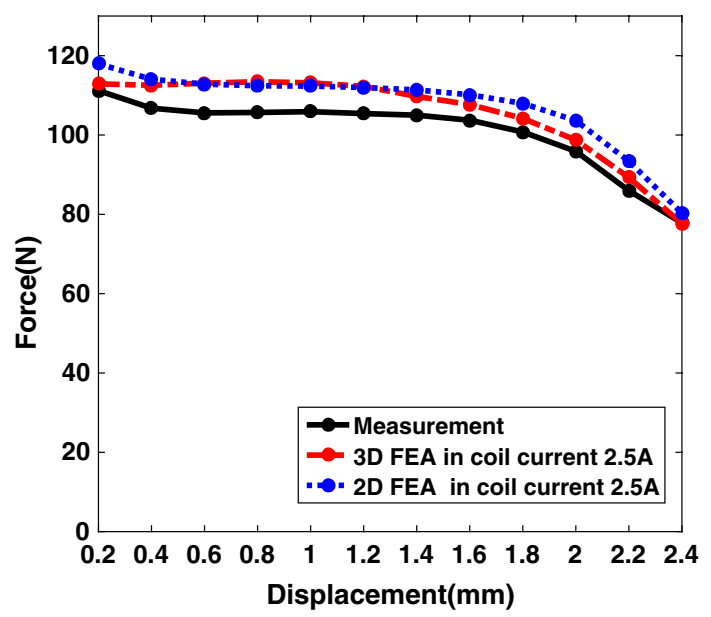

Fig.3 Static force versus displacement curve comparison about measurement, 3D FEA model and 2D FEA model

\subsection{Multi-objective problem description}

The maximum working stroke of LPS is $2 \mathrm{~mm}$ and sampling interval is set as $0.2 \mathrm{~mm}$. By using the FEA models in ANSYS MAXWELL software, the numerical calculation of static electromagnetic force is established at each sampling displacement. For LPS multi-objective problem, it can be explained by three objectives as follows:

(1) Average electromagnetic force $-\bar{F}$

The average electromagnetic force is calculated by each sampling point force. It is used to evaluate the excitation force performance in coil rated current.

(2) Standard deviation of static electromagnetic force $-F_{\sigma}$

The standard deviation of electromagnetic force is used to evaluate the nonlinearity degree of static electromagnetic force and coil current in working stroke.
(3) The average electromagnetic force-to-mass ratio of moving parts $-\bar{F} / m$

The mass of armature is the objective of LPS, and it is determined by the shape design parameters. And force-tomass ratio is one of the LPS dynamic performance evaluating indicator.

These objectives clearly evaluated the LPS shape design performance. Hence, the influence of each LPS shape design parameters should be qualitative analyzed before multi-objective optimization. The changing influence on $\bar{F}, F_{\sigma}$ and $\bar{F} / m$ is shown separately in Figs. 4, 5 and 6. All parameter variations are based on the value of conventional shape LPS. For example, in y-axis of Fig. 4, the items denote the variables of LPS design parameter by $+10 \%$ changing rate or $-10 \%$ changing rate. The $x$-axis of Fig. 4 shows the influence of each single variable in $\pm 10 \%$ changing rate. These comparisons in Figs.4, 5, 6 are using 2D FEA simulation result. In these figure, The parameter $\mathrm{Hc}, \mathrm{Ld} 1$ and $\mathrm{Rb}$ can be considered as most influential parameters of $\bar{F}$, while $\mathrm{Ld} 1, \mathrm{Hc}, \mathrm{Ld} 2$ as most influential parameters of $F_{\sigma}$ and $\mathrm{Hc}, \mathrm{Ha}, \mathrm{Ld} 1$ as most influential parameters of force-to-mass ratio. All the parameter changes are nonlinear relationship with $\bar{F}, F_{\sigma}$ and the forceto-mass ratio. Therefore, normal parameterization design method hardly gets an optimal LPS shape. This paper employed the validated 2D FEA model and employed a multi-objective LPS parametric optimization process,

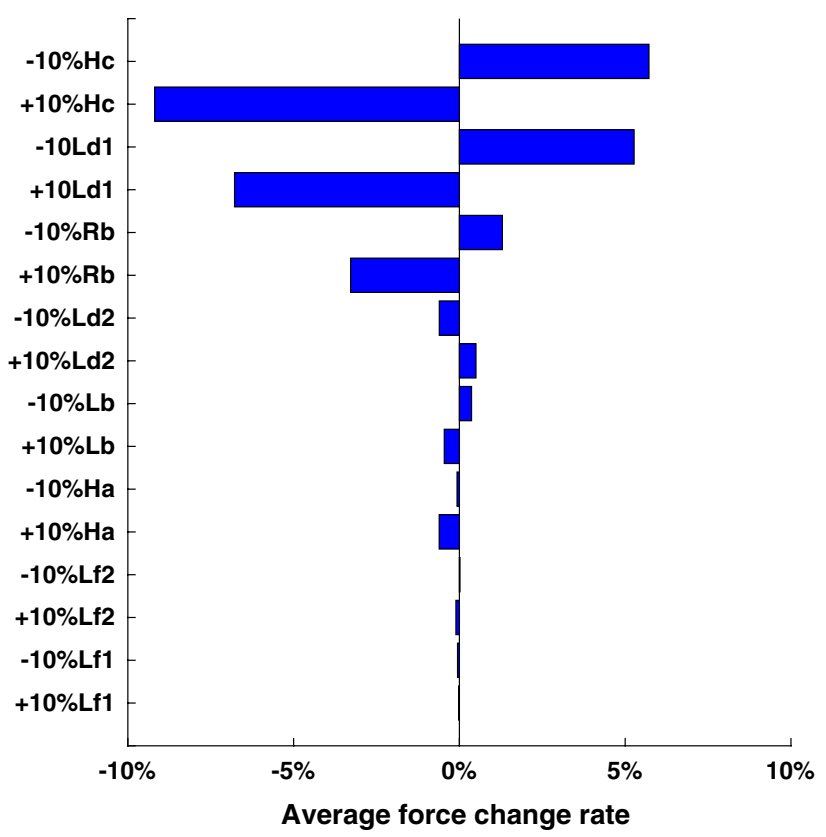

Fig.4 Average force change rate of each parameter based on the conventional shape LPS 


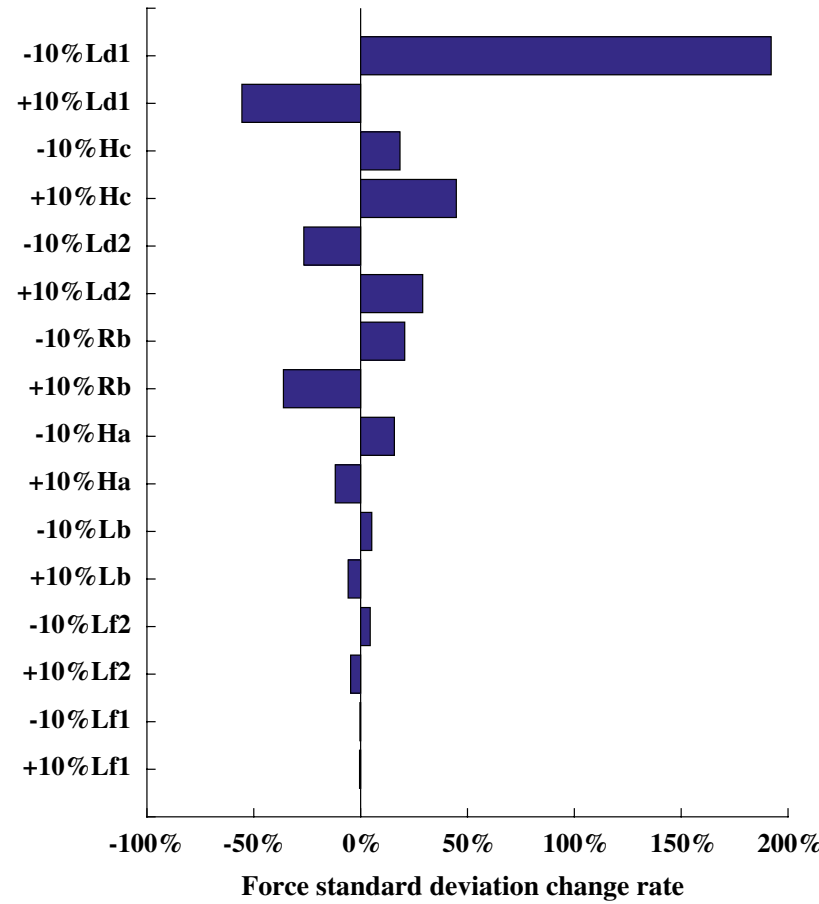

Fig.5 Force standard deviation change rate of each parameter

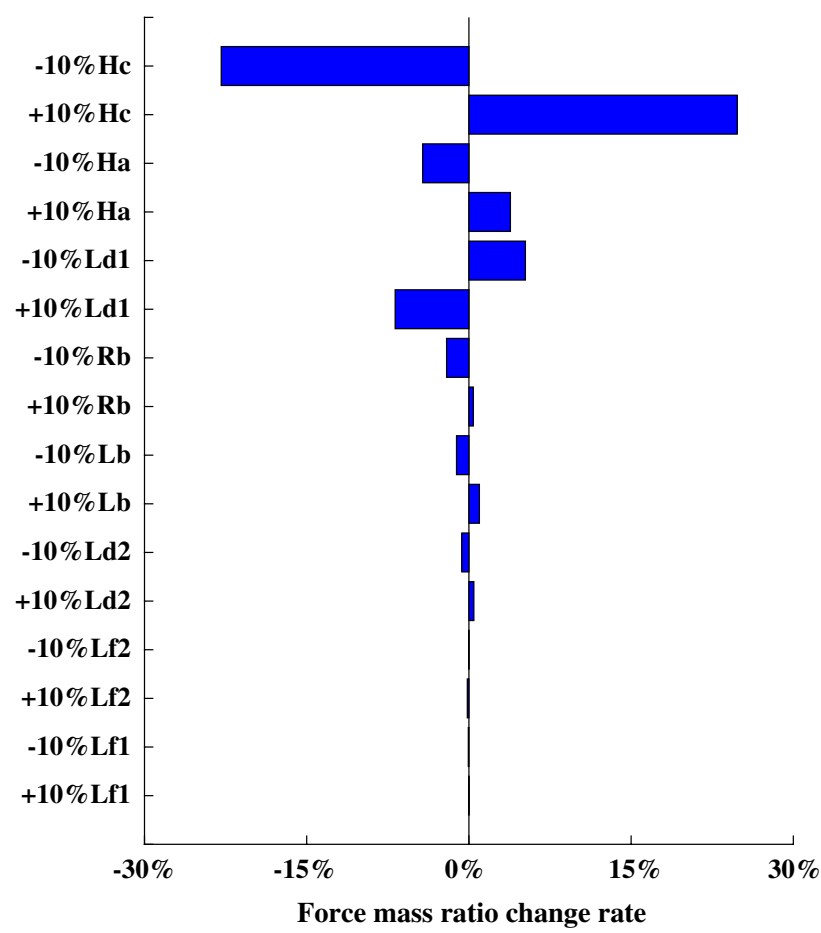

Fig.6 Force-to-mass ratio changing rate of each parameter based on the conventional shape LPS which includes GA parametric optimization and LPS output force magnetostatic numerical calculation.

To obtain normalized $\bar{F}, F_{\sigma}$ and force-to-mass ratio, the multi-objective fitness function of optimization can be described as follows:

fitness $=g_{1} \frac{\bar{F}_{\mathrm{ex}}-\bar{F}}{\bar{F}_{\mathrm{ex}}}+g_{2} \frac{F_{\sigma}}{\bar{F}}+g_{3} \frac{K_{\mathrm{ex}}-\bar{F} / m}{\bar{F}_{\mathrm{ex}}}$

Base on the fitness function, multi-objective design problem can be converted to the minimization cost problem in formula (13), where $g_{1}=1.1, g_{2}=1$ and $g_{3}=1.4$ are normalized factor. $\bar{F}_{\mathrm{ex}}$ is the expectation force, and $K_{\mathrm{ex}}$ is the expectation force-to-mass ratio. In this paper, the $\bar{F}_{e x}$ is set to $160 \mathrm{~N}$ and the $K_{\mathrm{ex}}$ is set to 3 .

\subsection{Genetic algorithm and ANSYS MAXWELL FEA simulation model description}

GA optimization tool in ANSYS MAXWELL is used to obtain the optimal LPS shape. And GA optimization process includes initialization, selection, genetic operators and Pareto front selection. The shape design parameters and values can be encoded as genes in chromosomes. And the optimization design parameters of the GA method are shown in Table 3 . The variables of initial individual are set as conventional values. After 20 Generations iteration, the GA optimization individuals are shown in Fig. 7.

After 20 generations of GA optimization process, an optimal result is obtained. The static force versus displacement curve of the result is shown in Fig. 8, and the values of design variables are shown in Table 4.

The comparison between the measurement and the simulation result underrated coil current (total 1188 A-turns) are shown in Fig. 8. The experimental results can verify the validity of the optimization approach by FEM. The maximum relative error between measurement and simulation is less than $16 \%$, in LPS working stroke from 0.4 to $1.4 \mathrm{~mm}$. Under this working stroke, the static force curve of the optimal result maintained a good constant value. From average

Table 3 GA optimization design parameters

\begin{tabular}{ll}
\hline GA design parameters & Value \\
\hline Number of individuals & 100 \\
Roulette selection & 5 \\
Total crossover type & Simulated binary \\
Crossover probability & 0.4 \\
Mutation type & Guass distribution \\
Total mutation probability & 0.25 \\
Number of survivors in Pareto front & 15 \\
Maximum number of iterations & 20 \\
\hline
\end{tabular}




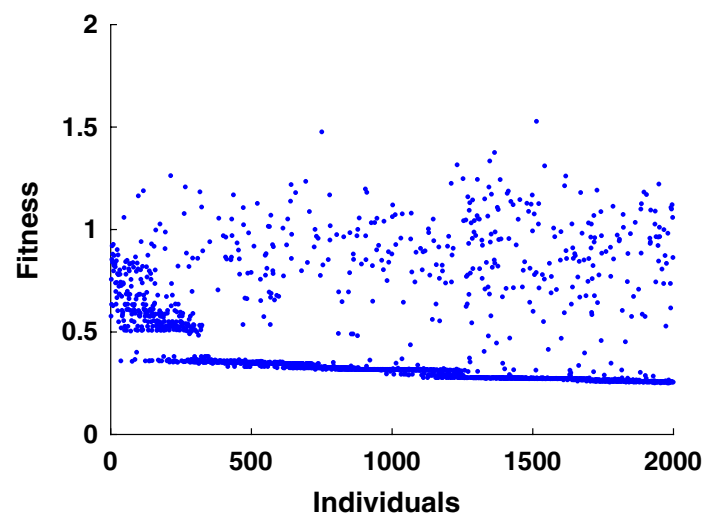

Fig.7 Individuals of GA optimization

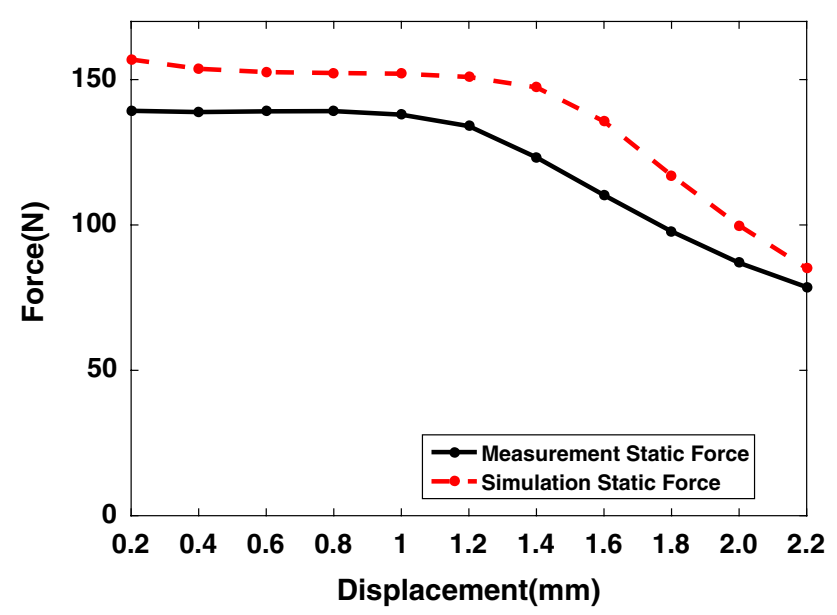

Fig.8 Static force versus displacement curve comparison of parameters LPS exciting current $5.4 \mathrm{~A}$

Table 4 Comparison of conventional values and optimal values
Table 5 Coil parameters of conventional LPS and optimal LPS

\begin{tabular}{lll}
\hline Coil parameters & Conventional & Optimal \\
\hline Resistance & $2.7 \Omega$ & $0.9 \Omega$ \\
Number of winding & 495 turns & 220 turns \\
Wire diameter & $0.63 \mathrm{~mm}$ & $0.83 \mathrm{~mm}$ \\
Rated current & $2.4 \mathrm{~A}$ & $5.4 \mathrm{~A}$ \\
\hline
\end{tabular}

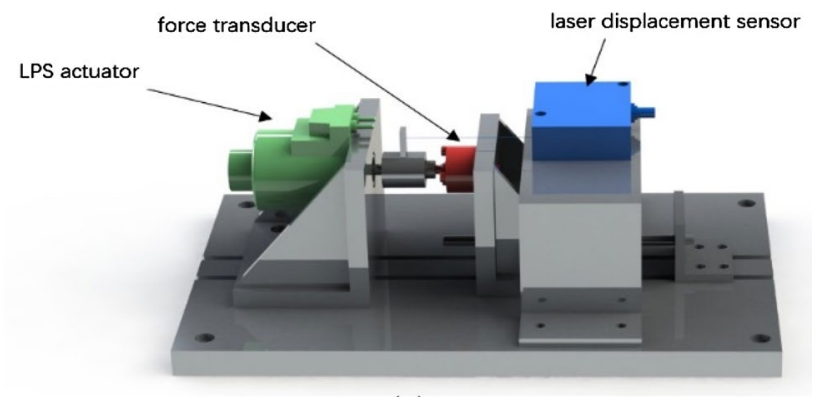

(a)

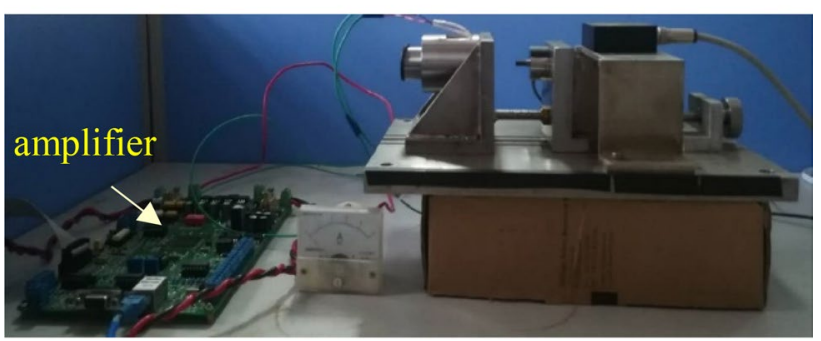

(b)

Fig.9 (a) LPS and the testing devices. (b) LPS dynamic and static performance test

force point of view, the measurement comparison result between optimal shape and conventional shape changed from 106.5 to $138.6 \mathrm{~N}$, which increased about $30.1 \%$ in total. From standard deviation of force point of view, the measurement comparison result changed from 1.98 to $2.16 \mathrm{~N}$, which increased about $9.1 \%$ in total. From static force-to-mass ratio point of view, the measurement comparison result changed from 2.31 to 2.48 , which increased about $7.6 \%$ on the whole.

\section{Comparison studies}

The conventional structure LPS and optimized structure prototype are manufactured. The parameters of manufactured coils are shown in Table 5. To ensure the accuracy of measurement results, ZLDS100 (ZSY GROUP LTD) laser displacement sensor and U9C (HBM) force transducer are employed in LPS's static and dynamic performance tests. And a 1MS/s, 16-channels analog inputs, 16-bit resolution multifunction DAQ Card(ADVANTECH) is employed to 
given coil current order to LPS amplifier, and sampling the coil current signal, push force signal and displacement signal. LPS performance test device is shown in Fig. 9.

The static performance comparison of optimal shape and conventional shape is obtained as shown in Fig. 10a. The static force versus displacement curves indicate that the optimal shape LPS has good linearity as conventional type in working stroke and the static force. And static force versus Ampere-turn test result in Fig. 10b presented the static magnetic force of optimal shape large than conventional shape in each Ampere-turn at whole working stroke position. Start position $(1.4 \mathrm{~mm})$ and middle position $(0.9 \mathrm{~mm})$ and end position $(0.4 \mathrm{~mm})$ are selected to illustrate these results, respectively. For both the static force of conventional type and optimal type in Fig. 10a and b, the improvement in static force is distinguishable for optimal LPS.

Figure 11 illustrates the dynamic response performance of electromagnetic force of the LPS at a fixed position. Since the phase frequency characteristics of the force are better than the amplitude frequency characteristics, the amplitude frequency characteristic curve is only shown in this figure. In

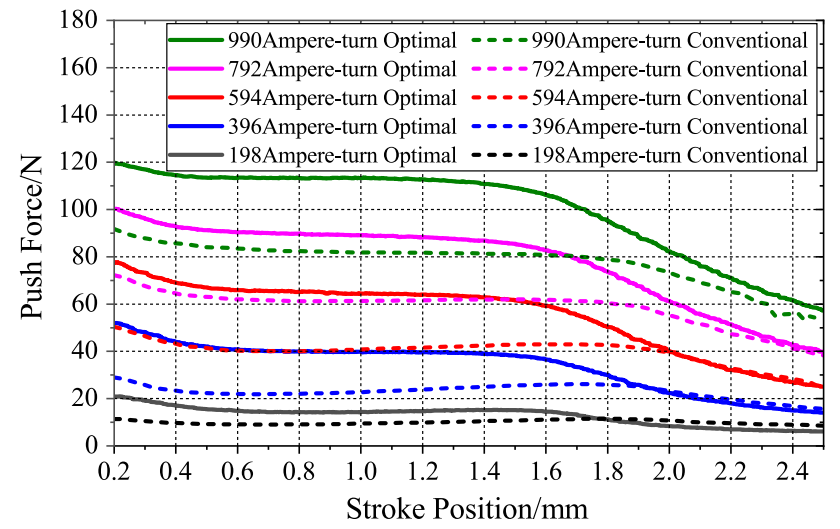

(a)

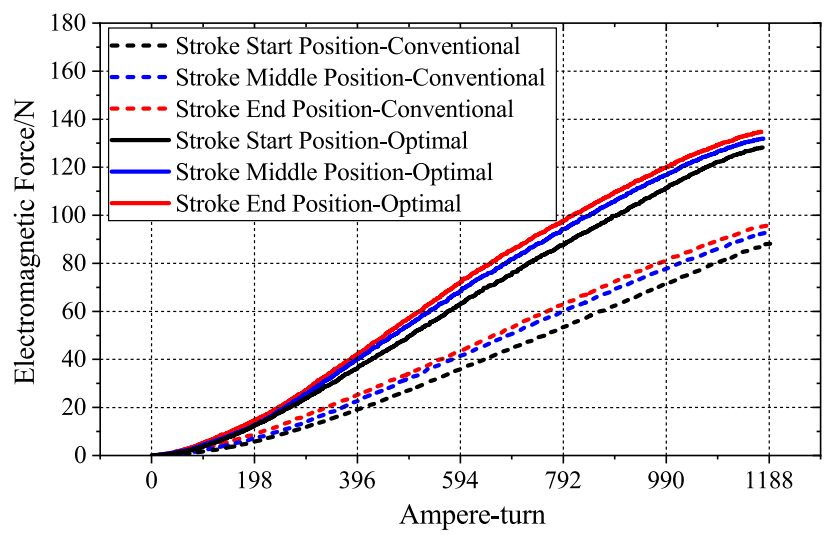

(b)

Fig.10 (a) Static force versus displacement test and (b) static force versus Ampere-turn test

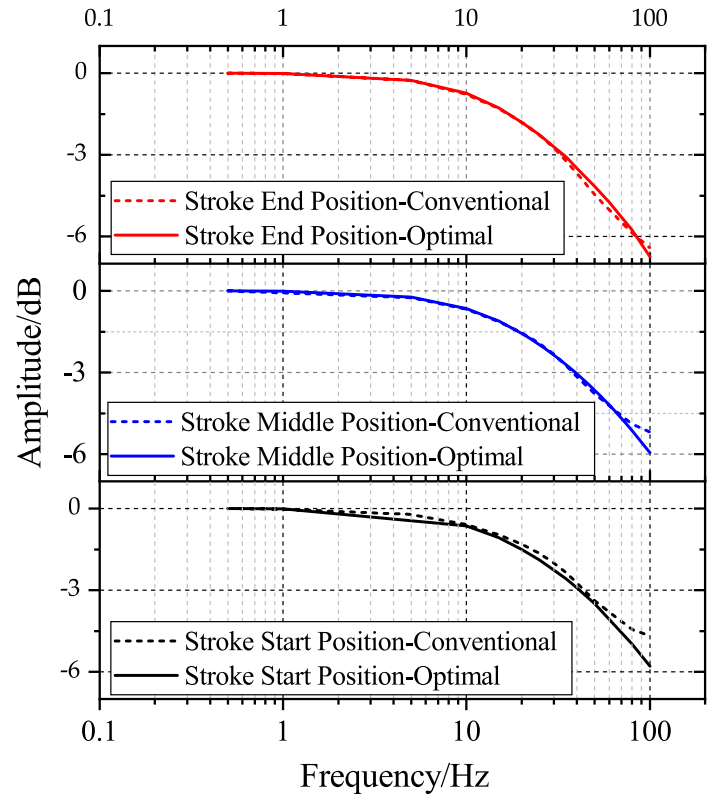

Fig.11 Dynamic force frequency response at fixed position

comparison with conventional shape, the results indicate that the dynamic frequency response of the electromagnetic force of the optimal shape is not significantly improved under the same magnetic material (electromagnetic pure iron). When the displacement increases, the frequency response will increase accordingly. The amplitude frequency response is increased from $35 \mathrm{~Hz}$ at the end position of stroke $(0.4 \mathrm{~mm})$ to $40 \mathrm{~Hz}$ at the start position of stroke $(1.4 \mathrm{~mm})$.

The difference in dynamic performance between the optimized LPS and the conventional LPS is illustrated in Fig. 12, by the coil current and push force step response at the middle position of working stroke $(0.9 \mathrm{~mm})$. The measurement result of step response in rated coil current (1188 A-turn) is shown in Fig. 12a. The rise time in Table 6 is the variables rise from 10 to $90 \%$, and it indicates that the coil current rise time can be reduced by $55.6 \%$ and the rise time of push force is reduced by $6.8 \%$. The step response of the falling edge shown in Fig. 12b and the fall time in Table 6 are the variables fall from 90 to $10 \%$, the coil current fall time can be reduced by $34.5 \%$, and falling edge response is faster than rising edge due to the LPS amplifier's drive circuit. Hence, the push force fall time of optimal LPS is increased by $39.0 \%$. This phenomenon can be potentially explained for the large push force and quick current response.

The displacement step response is another dynamic performance validation approach, except force step response and frequency response at fixed position. The coil current input signal, coil actual current, LPS push force and armature displacement are measured by the testing devices. Tables 7 and 8 present the comparison between optimal LPS and conventional LPS transient step response, 


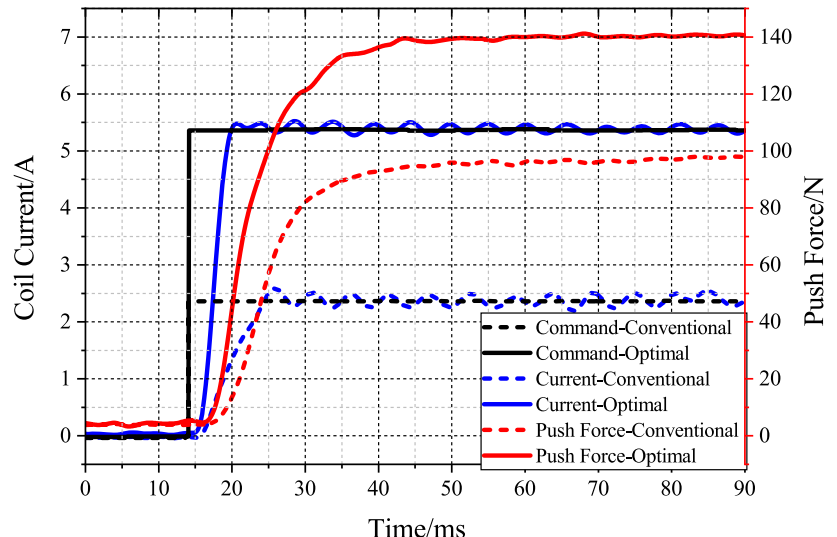

(a)

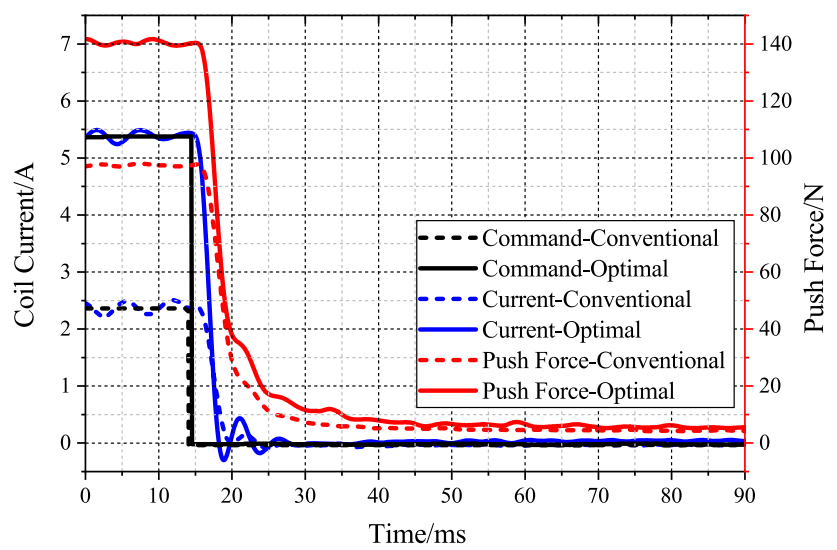

(b)

Fig.12 (a) Rising edge of force step response at stroke middle position and (b) falling edge of force step response at stroke middle position

Table 6 Force step response at stroke middle position

\begin{tabular}{lll}
\hline Performance & Conventional LPS & Optimal LPS \\
\hline Coil current rise time & $7.2 \mathrm{~ms}$ & $3.2 \mathrm{~ms}$ \\
Push force rise time & $14.6 \mathrm{~ms}$ & $13.6 \mathrm{~ms}$ \\
Coil current fall time & $2.9 \mathrm{~ms}$ & $1.9 \mathrm{~ms}$ \\
Push force fall time & $8.2 \mathrm{~ms}$ & $11.4 \mathrm{~ms}$ \\
\hline
\end{tabular}

Table 7 Transient step response in spring stiffness 10400 N/mm

\begin{tabular}{lll}
\hline Performance & Conventional LPS & Optimal LPS \\
\hline Displacement rise time & $3.8 \mathrm{~ms}$ & $3.6 \mathrm{~ms}$ \\
Push force rise time & $3.9 \mathrm{~ms}$ & $3.1 \mathrm{~ms}$ \\
\hline
\end{tabular}

meanwhile the details are shown, respectively, in Figs. 13 and 14 . Under different spring stiffness $(10400 \mathrm{~N} / \mathrm{m}$ and
Table 8 Transient step response in spring stiffness 32900 N/mm

\begin{tabular}{lll}
\hline Performance & Conventional LPS & Optimal LPS \\
\hline Displacement rise time & $15.9 \mathrm{~ms}$ & $9.0 \mathrm{~ms}$ \\
Push force rise time & $15.7 \mathrm{~ms}$ & $8.7 \mathrm{~ms}$ \\
\hline
\end{tabular}

$32900 \mathrm{~N} / \mathrm{m}$ ) and $1.5 \mathrm{~mm}$ spring pre-compression operating condition, the displacement step response rise time of optimal LPS is reduced $5.2 \%$ and $43.4 \%$, and force step response rise time is reduced $20.5 \%$ and $44.6 \%$. Consequently, the optimization method proposed in this paper can simultaneously improve the static force and dynamic response of the electromagnet. The optimized LPS has a better performance, particularly in high push force and quick motion usage.

\section{Conclusions}

This paper presents a shape design optimization process of LPS, by using the GA method and magnetostatic force 2D FEA method. It aims to optimize the static and dynamic LPS performance through its shape design and rated current.

According to the actual usage in this research, the applicable coil structure parameters are proposed and the multiobjective GA optimization process is adopted to optimize the static force in working stroke. The accuracy of 2D FEA model is validated by the comparison of 3D FEA and the measurement. In addition, the optimization results demonstrate that the whole test could spend less time on the process by using 2D FEA and the 2D FEA has the equal accuracy as 3D FEA in the test; meanwhile, the errors reflected from the experimental work are acceptable.

Through using the proposed GA optimization method in this paper, an optimal LPS shape parameters result is obtained and this optimal LPS shape is manufactured. A series of experimental tests are conducted to validate the static performance and dynamic performance. By the comparison of conventional shape LPS, the proposed optimal shape average static force in normal working stroke is $138.6 \mathrm{~N}$, and it has improved $30.1 \%$ by the comparison of conventional shape. The optimal shape current rise time and fall time have been reduced, respectively, by $55.6 \%$ and $34.5 \%$, in the test of coil current step input response at fixed stroke position. Meanwhile, LPS displacement step response rise time has been reduced $5.2 \%$ and $43.4 \%$, and force step response rise time is reduced $20.5 \%$ and $44.6 \%$ by under different spring stiffness $(10400 \mathrm{~N} / \mathrm{m}$ and $32900 \mathrm{~N} / \mathrm{m}$ ) and $1.5 \mathrm{~mm}$ spring pre-compression operating condition. Above all, the multi-objective GA optimization 


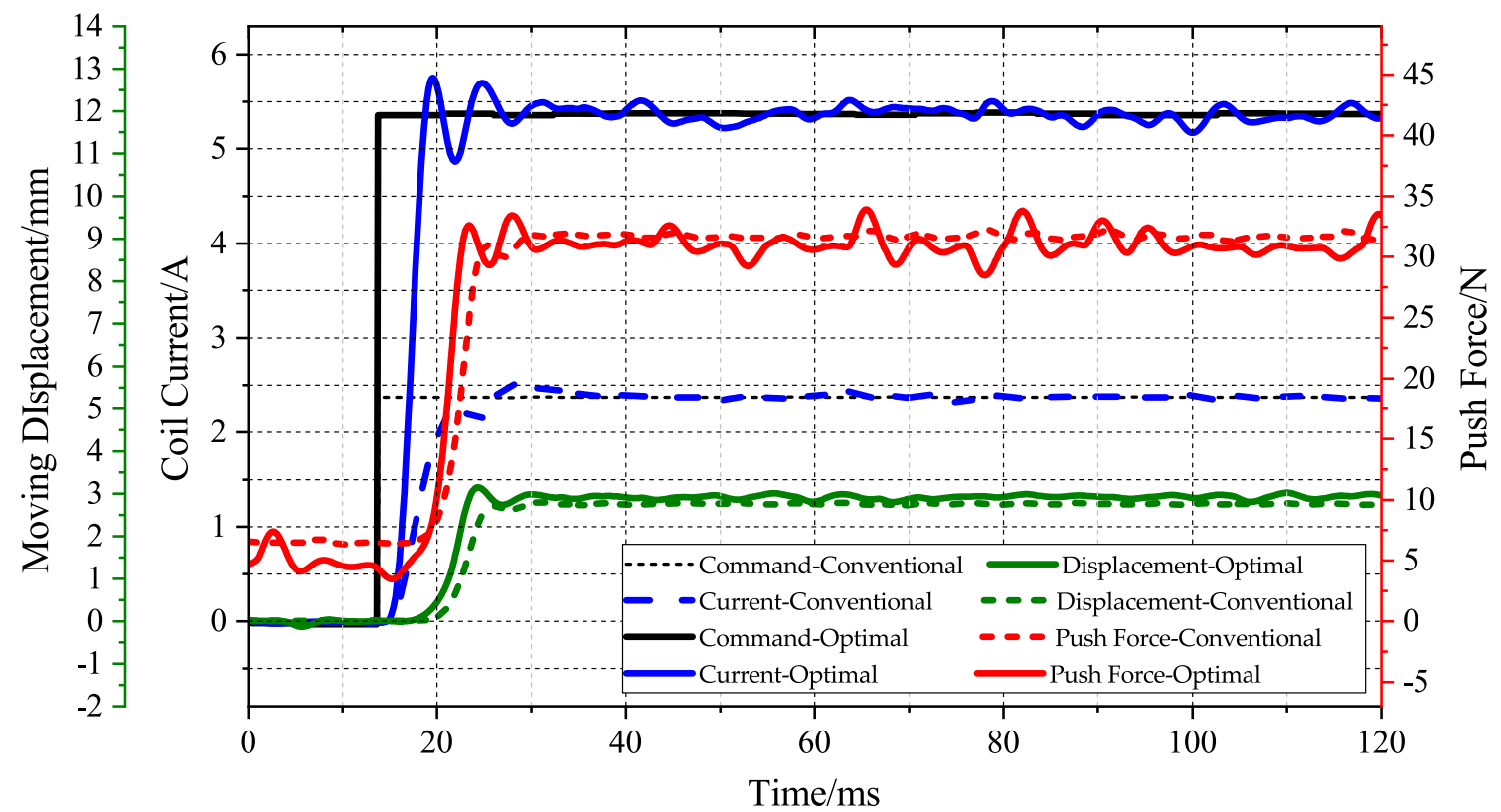

Fig.13 Displacement step response under spring stiffness 10,400 N/mm
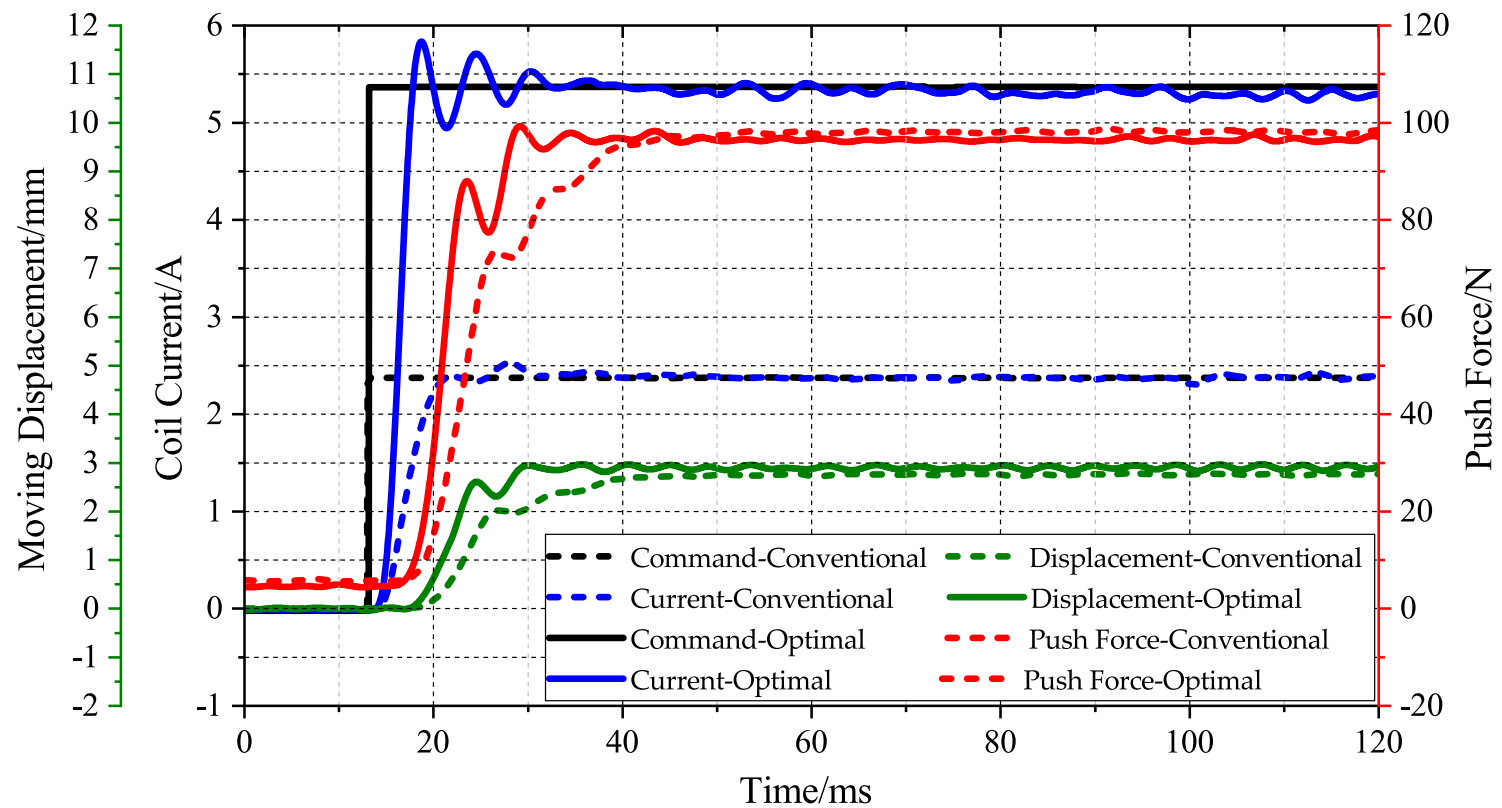

Fig.14 Displacement step response under spring stiffness 32,900 N/mm

of LPS's validity could be testified by the static and dynamic performance test devices.
Funding This research was funded by Ningbo Municipal Bureau of Science and Technology, grant number 2019B10052, and National Natural Science Foundation of China, Grant No. 51521064. 


\section{Compliance with ethical standards}

Conflict of interest The authors declare that they have no conflict of interest.

Open Access This article is licensed under a Creative Commons Attribution 4.0 International License, which permits use, sharing, adaptation, distribution and reproduction in any medium or format, as long as you give appropriate credit to the original author(s) and the source, provide a link to the Creative Commons licence, and indicate if changes were made. The images or other third party material in this article are included in the article's Creative Commons licence, unless indicated otherwise in a credit line to the material. If material is not included in the article's Creative Commons licence and your intended use is not permitted by statutory regulation or exceeds the permitted use, you will need to obtain permission directly from the copyright holder. To view a copy of this licence, visit http://creativecommons.org/licenses/by/4.0/.

\section{References}

1. Lee SH, Yi HC, Han K, Kim JH (2015) Genetic algorithm-based design optimization of electromagnetic valve actuators in combustion engines. Energies 8:13222-13230

2. Lee HR, Ahn JH, Kim HY (2016) Design of a solenoid actuator for a cylinder valve in a fuel cell vehicle. Appl Sci 6:288

3. Zhao J, Yue P, Grekhov L, Ma X (2018) Hold current effects on the power losses of high-speed solenoid valve for common-rail injector. Appl Therm Eng 128:1579-1587

4. Cai B, Liu Y, Tian X, Wang Z, Ji R (2010) Optimization of submersible solenoid valves for subsea blowout preventers. IEEE Trans Magn 47(2):451-458

5. Wu S, Zhao X, Li C, Jiao Z, Qu Y (2017) Multi-objective optimization of a hollow plunger type solenoid for high speed on/off valve. IEEE Trans Ind Electron 65(4):3115-3124
6. Yang YP, Liu JJ, Ye DH, Chen YR, Lu PH (2013) Multiobjective optimal design and soft landing control of an electromagnetic valve actuator for a camless engine. I IEEE-ASME Trans Mechatron 18(3):963-972

7. Wang L, Li GX, Xu CL, Xi X, Wu XJ, Sun SP (2016) Effect of characteristic parameters on the magnetic properties of solenoid valve for high-pressure common rail diesel engine. Energy Convers Manag 127:656-666

8. Yun SN, Ham YB, Park JH (2012) New approach to design control cone for electro-magnetic proportional solenoid actuator. In: IEEE/ASME international conference on advanced intelligent mechatronics. IEEE. https://doi.org/https://doi.org/10.1109/ AIM.2012.6265943

9. Park CD, Lim BJ, Chung KY (2015) Design verification methodology for a solenoid valve for industrial applications. J Mech Sci Technol 29(2):677-686

10. Yamada H, Kihara S, Yamaguchi M, Nakagawa H, Hagiwara $K$ (1994) Static thrust improvement of a linear proportional solenoid. J Magn Soc Jpn 9(6):117-121

11. Shin Y, Lee S, Choi C (2015) Shape optimization to minimize the response time of direct-acting solenoid valve. J Magn 20(2):193-200

12. Liu P, Fan L, Zhou W, Ma X, Song E (2017) Dynamic performances analysis and optimization of novel high-speed electromagnetic actuator for electronic fuel injection system of diesel engine. J Mech Sci Technol 31(8):4019-4028

13. Plavec E (2016) Genetic algorithm based plunger shape optimization of DC solenoid electromagnetic actuator. IEEE TELFOR 2016. IEEE. https://doi.org/https://doi.org/10.1109/TELFO R.2016.7818839

Publisher's Note Springer Nature remains neutral with regard to jurisdictional claims in published maps and institutional affiliations. 